\title{
Civilisations
}

Revue internationale d'anthropologie et de sciences

humaines

48 | 2001

La question de l'islam et de l'Etat à l'aube du XXle siècle

\section{Islam, état, laïcité : le cas de la Turquie dans sa perspective historique}

L'avènement du régime kémaliste et la rupture avec l'ordre islamique

Robert Anciaux

\section{CpenEdition}

Journals

Édition électronique

URL : http://journals.openedition.org/civilisations/3456

DOI : $10.4000 /$ civilisations.3456

ISSN : 2032-0442

Éditeur

Institut de sociologie de l'Université Libre de Bruxelles

Édition imprimée

Date de publication : 31 août 2001

Pagination : 27-50

ISSN : 0009-8140

Référence électronique

Robert Anciaux, «Islam, état, laïcité : le cas de la Turquie dans sa perspective historique », Civilisations [En ligne], 48 | 2001, mis en ligne le 01 août 2004, consulté le 21 décembre 2020. URL : http:// journals.openedition.org/civilisations/3456; DOI : https://doi.org/10.4000/civilisations.3456

(c) Tous droits réservés 


\section{ISLAM, ÉTAT, LAICITE : \\ LE CAS DE LA TURQUIE DANS SA PERSPECTIVE \\ HISTORIQUE \\ L'AVÈNEMENT DU RÉGIME KÉMALISTE \\ ET LA RUPTURE AVEC L'ORDRE ISLAMIQUE}

\section{Robert ANCIAUX}

\section{INTRODUCTION}

Dans le courant des années 1920, Mustafa Kemal Atatürk et ses partisans mettaient en œuvre une série de réformes radicales : suppression du sultanat, abolition du khalifat, abrogation de la sharia et adoption de codes de lois européens, suppression de la mention de l'slam comme religion de l'État turc dans la constitution, adoption de l'alphabet latin au détriment de l'alphabet arabe. Ces bouleversements visaient à influer de manière décisive sur la nature de l'État et de la société turcs appelés à se situer dans le prolongement de la civilisation occidentale scientifiquement et technologiquement développée. Sur le terrain de l'occidentalisation, étape jugée préalable à la sortie de l'état de sous-développement, le régime kémaliste développa une vision globalisante qui l'isolait des autres régimes réformistes même les plus modernisateurs, du monde musulman. De fait, la Turquie opérait une rupture volontairement radicale avec ses racines musulmanes. Par les finalités exclusivement séculières qu'elle s'assignait la nouvelle république turque rejetait catégoriquement celles qui fondaient la cohérence de l'État islamique.

Celui-ci, qui commença à s'édifier dès lors que la communauté musulmane se constitua en entité politique, se définit, dès le départ, en fonction des prescriptions de la révélation transmise par Dieu à son Prophète, Muhammad.

L'État islamique aura ainsi pour fonction essentielle d'offrir aux croyants le cadre institutionnel de nature à favoriser l'accomplissement des finalités assignées par Dieu à sa communauté. La mission ainsi prescrite aux musulmans est d'assurer, par leurs actes et leurs comportements, la reproduction sur terre de la cité de Dieu, archétype de l'ordre juste et parfait dans lequel chacun sera rétribué selon ses besoins et selon ses mérites.

L'État islamique selon la définition qu'en donne le penseur et idéologue réformiste Rachid Rida (m. 1935) est présenté comme la structure politique qui «réunit la justice, l'égalité, fait respecter les intérêts de chacun, empêche les maux, ordonne le bien, interdit le mal, veille sur les mineurs, les infirmes, les invalides, secourt les pauvres et les indigents grâce à l' aumône que les musulmans doivent payer...»' .

Dans sa conception initiale l'islam apparaît comme un projet de société explicite et comme l'inspiration d'un projet politique, non explicitement défini, fondés sur les notions essentielles de fraternité et de solidarité entre croyants unis par la poursuite d'un but ultime commun. $\mathrm{La}$ 
religion constitue ainsi, le pôle identitaire qui assure la cohésion de la cité musulmane, et par lequel les membres du corps social ont conscience d'appartenir à un ensemble cohérent.

Dans la mesure où son existence en tant qu'entité politique singulière était liée à sa fonction d'exécutant des desseins de Dieu, exerçant une souveraineté sans partage sur l'ensemble de sa création, l'État islamique se dota d'un cadre institutionnel en rapport avec sa nature. La sharia, ensembles des règles de vie spécifiquement islamiques dans leurs différentes versions sunnites tirées de l'exégèse du Coran et de la Sunna, ainsi que de la déduction par analogie, fournit le cadre de référence juridique, tandis que la réflexion de théoriciens sunnites - tels, entre autres Mawârdî ou Ibn Taymiyya - et shiites - comme Nasîr al-Dîn Tûsî - contribua à la définition de cadres institutionnels conformes à la nature d'une entité politique islamique.

D'une manière générale, dès qu'il fut pourvu à la succession du Prophète, le chef de l'État musulman - à l'exception des Imâm(s) shiites descendants de Muhammad par Alî et Fâtima a disposé d'un pouvoir exclusivement exécutif. Ni le khalife, ni aucun autre chef d'État sunnite, ni shiite depuis la ghayba (occultation), ne peut être considéré comme investi d'un pouvoir religieux. Certes, les Abbassides ont tenté d'imposer la notion de quasi pouvoir de droit divin, en vain, De son côté, dans le souci de justifier l'existence d'un khalifat abbasside dépossédé de tout pouvoir effectif par ses grands vassaux, Al-Ghazzâli va insister sur le caractère emblématique du khalife qui doit se poser en figure exemplaire de l'islam par l'étendue de ses connaissances en matière de sciences religieuses et l'intensité de sa piété. Ceci ne suffira toutefois pas à faire du khalife, ou de tout autre souverain musulman, le détenteur d'un quelconque pouvoir religieux.

Sur ce plan nous nous rallierons donc à la proposition formulée par le shaykh Muhammad Abduh selon lequel : «L'un des plus grands et des plus nobles principes que l'islam ait proclamés est l'abolition du pouvoir religieux... Il n'existe aucunement en islam ce que certains appellent pouvoir religieux» ${ }^{2}$.

Cette approche du pouvoir religieux est encore accentuée par Alî Abd al-Râzik, dont la conception du pouvoir et de la religion provoqua, il est vrai, une réaction virulente de la majorité des théologiens-juristes conservateurs de l'époque. La religion musulmane, écrit-il, «est innocente de cette fonction califale telle que l'entendent les musulmans... Celle-ci n'a rien de commun avec les fonctions religieuses, non plus que la judicature et les autres fonctions et rouages essentiels du pouvoir et de l'État. Toutes ces fonctions sont purement politiques et la religion ne les a ni reconnues ni remises» ${ }^{3}$.

Toutefois, si l'islam n' a pas prévu de pouvoir religieux, le khalife, ou tout autre gouvernant musulman, se voit investi du devoir de défendre et de promouvoir la religion par ses attitudes et ses actes. Il se doit ainsi d'être un personnage exemplaire par sa piété et son respect de la Loi. La légitimité de son pouvoir repose essentiellement sur sa capacité à défendre l'islam contre l'ennemi extérieur et intérieur, et à veiller à l'application de la Loi sur l'ensemble du territoire qu'il contrôle. Le devoir essentiel d'un gouvernant musulman est, selon les termes utilisés par al Ghazâlî dans le Kitâb al Iqtisâd et le Kitâb al Mustazhirî, de veiller à instaurer le «bon ordre du monde» fondement nécessaire à l'établissement du «bon ordre de la religion».

Le devoir religieux que recouvre l'acte de gouverner les hommes est aussi abondamment souligné par le théologien-juriste hanbalite Ibn Taymiyya, dont l'activité s'étendit sur le dernier quart du XIII' et le premier quart du XIV ${ }^{e}$ siècle. Gouverner les affaires des hommes, écrit-il, «est l'une des exigences les plus importantes de la religion et, même plus sans gouvernement 
la religion ne peut subsister... Un dirigeant est nécessaire à la vie en société... Le devoir de commander le bien et d'interdire le mal ne peut être accompli sans pouvoir et sans autorité... L'exercice du pouvoir est une tâche religieuse et une bonne action qui rapproche de Dieu» ${ }^{4}$.

Récusant le caractère nécessaire du khalifat, il reconnaît comme gouvernant légitime tout détenteur de l'autorité qui veille à l'application de la sharia et se conforme à ses prescriptions. Il cautionne ainsi tout gouvernement légaliste qui place son autorité au service de la réalisation des finalités prescrites à la communauté des croyants par la révélation divine. S'il n'y a donc pas confusion entre pouvoir religieux et pouvoir temporel comme le démontre, plus que toute autre la démarche de Ibn Taymiyya, il y a bien, au niveau des finalités assignées aux sociétés humaines, subordination du gouvernement des hommes aux prescriptions de la révélation. Le légalisme prôné par Ibn Taymiyya constitua l'un des éléments essentiels sur lesquels les Ottomans fondèrent leur légitimité.

A partir de la fin du XVIII ${ }^{e}$ siècle pour compenser la perte de prestige que leur valut la perte de territoires au profit de la Russie, le sultan ottoman, se voit reconnaître, à titre de compensation morale, par le vainqueur, une sorte d'autorité spirituelle transfrontalière sur les populations musulmanes soustraites à son autorité. Le sultan ottoman, dès lors, va revendiquer pour lui le titre de khalife qui selon, une légende récente, aurait été transmis, en 1517, à son ancêtre Sélim $\mathrm{I}^{\mathrm{er}}$ par le dernier khalife abbasside résidant au Caire. «La revendication par la sultan ottoman», écrit Bernard Lewis, «d'une sorte de pontificat religieux s'étendant aux musulmans autres que ses propres sujets était nouvelle et sans précédent. Depuis l'extinction du khalifat islamique classique au Moyen Âge, il n'y a pas eu de titulaire universellement reconnu à la tête de la communauté musulmane... Depuis la chute des Abbassides c'était la première tentative de rétablir un leadership islamique universel, et de le réclamer pour la Maison d'Osman» ${ }^{5}$. Cette vision d'un leadership spirituel s'imposant au monde musulman, que ne conforte ni la doctrine ni la pratique traditionnelle en islam, fut néanmoins reprise en Occident où le khalifat apparut comme un titre qui cumulait pouvoir spirituel et temporel. Interprétation que réfuta énergiquement, comme nous l'avons déjà mentionné, le shaykh d'al-Azhar, Muhammad Abduh.

Toutefois, les prétentions des sultans ottomans à un pontificat islamique suprême vont trouver quelques échos, en dehors des frontières de l'Empire, auprès des populations musulmanes sunnites dominées par les puissances européennes. Tandis que, tout au long du XIX $\mathrm{X}^{\mathrm{e}}$ siècle, des pans de plus en plus large du monde musulman sont subjugués par des États en pleine expansion, l'Empire ottoman apparaît comme l'une des rares entités musulmanes encore indépendante et disposant d'une relative puissance militaire. C'est donc vers lui que se tournent de plus en plus nombreuses, les populations musulmanes occupées par des forces occidentales. Il n'est, dès lors, pas difficile pour les sultans-khalifes Abd ül-Medjid et Abd ül-Hamid de présenter l'Empire ottoman comme la qibla de l'Islam et de lancer un appel à l'unité de tous les musulmans autour de la personne du khalife pour faire pièce à l'expansion européenne. Cette propagande panislamiste dont les sultans attendaient qu'elle leur apporte des appuis extérieurs dans leur tentative de freiner l'avancée occidentale, si elle rencontra un certain succès auprès des masses dans le monde musulman n'empêcha pas le grignotage de l'Empire par les grandes puissances. Mieux même, l'idéologie nationaliste gagnant du terrain parmi les populations musulmanes non-turques, celles-ci, rebutées par les manifestations récentes d'un nationalisme turc hégémonique, restant sourdes aux appels au jihâd du sultan, se joignirent aux Alliés durant la première guerre mondiale dans l'espoir de voir satisfaites leurs revendications nationales. 
Néanmoins, au lendemain de la Première Guerre mondiale, la plupart des pays musulmans occupés par une puissance européenne, se tournent vers le khalifat comme seul pôle de référence religieuse commun et le seul repère symbolique de l'unité spirituelle de l'ensemble du monde musulman organiquement divisé et politiquement soumis à différentes autorités étrangères non-musulmanes. Aussi, l'abolition du khalifat par les autorités kémalistes plongeat-elle l'ensemble des musulmans dans un profond désarroi dont il fut long à se remettre. Cette mesure fut à l'origine d'une rancœur et d'une méfiance persistante du monde musulman à l'égard de la république turque.

Au plan local turc, quels qu'aient été, toutefois, les développements théoriques que connut l'exercice du pouvoir dans l'Empire ottoman, celui-ci, jusqu'à la fin de la Première Guerre mondiale, restait fondamentalement un cadre politique conçu pour une société musulmane; c'est-à-dire pour une population fondamentalement attachée, malgré les innovations juridicoadministratives introduites au XIX ${ }^{\mathbf{e}}$ siècle, à une Loi qui n'a pas pour finalité essentielle de réglementer les rapports sociaux de manière à créer un ordre fonctionnel des choses susceptible de permettre la vie en société, mais d'indiquer aux hommes les attitudes à adopter et les actes à poser pour réaliser les finalités fixes par une source révélée. Quant à l'État, nous l'avons déjà souligné, il a pour fonction première d'offrir un cadre politique où cette Loi est d'application pour tous les croyants soucieux d'œuvrer à la réalisation des finalités indiquées par la révélation. Et c'est bien ainsi qu'il était perçu par la majorité des populations musulmanes.

On mesure donc le bouleversement qu'a pu représenter, dans une société de traditions musulmanes, la nature du train de réformes mis en œuvre à l'initiative de Mustafa Kemal Atatürk, dans la mesure où, les concepts de nationalisme et de laïcité, qui apparaissent comme les piliers fondamentaux de l'idéologie kémaliste, impliquent une rupture radicale avec les fondements théologiques de l'État islamique. En effet, le principe de la souveraineté de la nation invoqué par les kémalistes, en référence à la conception de la souveraineté nationale développée par les nations européennes, implique la négation de la vision islamique de la souveraineté absolue de Dieu sur sa création. En outre, le concept de laïcité, principe essentiel définissant l'identité et la nature de la nation turque et considéré comme la condition première du progrès, visait «à créer une citoyenneté pour un individu libéré de la contrainte communautaire de l'umma, vivant dans une société contractuelle et démocratique, dans un espace public structuré par la raison et la science et non plus par la religion confinée dans l'espace privé» ${ }^{6}$. Les finalités exclusivement séculières assignées à la société turque entraient en contradiction flagrante avec la vision qu'avait la majorité de la population turque des finalités qu'elle était tenue de poursuivre en sa qualité de membre de la communauté musulmane. Il n'est donc pas surprenant qu'une partie importante du corps social turc ait considéré les réformes läques comme une véritable agression contre les systèmes de références culturelles et religieuses existants auxquels elle s'identifiait. Dès la naissance de la République on assistera donc à une opposition durable entre une grande partie de la population et les élites kémalistes, dont principalement l'establishment militaire identifié à l'origine comme le gardien des valeurs kémalistes. La voie de l'occidentalisation radicale suivie par Kemal Atatürk n'indispose d'ailleurs pas que les masses turques. Nombre de ses anciens compagnons d'arme pendant la guerre d'indépendance ne suivent pas sa vision globalisante de l'ouverture à la modernité, et fondent même un parti d'opposition : le Parti Républicain Progressiste. Le pluralisme politique étant considéré comme un frein à sa marche vers la modernité, Mustafa Kemal profitera de la révolte de 1925, pour y mettre un terme. Le thème central au nom duquel les kémalistes justifient et légitiment leur action est l'intérêt supérieur de l'État et de la nation. En vertu de cet 
impératif, la Turquie moderne s'est construite, au départ, contre le peuple et sans le peuple, tout entier mobilisé pour l'édification d'une nation moderne, souveraine et développée, objectif central de la révolution kémaliste.

\section{LA NATION TURQUE, UNE ET INDIVISIBLE, ET L'ISLAM}

Le nationalisme et la laïcité apparaissent comme les concepts centraux de l'idéologie kémaliste. Ce sont aussi ceux que la majorité des citoyens turcs auront le plus de mal à intégrer. Les réformes institutionnelles et l'édification d'un système d'enseignement d'inspiration européenne auront pour objet de créer un cadre favorable à l'implantation autoritaire de ces concepts dans la conscience collective du peuple turc.

Le nationalisme turc n'est pas le résultat de l'éveil d'une conscience nationale au sein de la population anatolienne, mais le produit d'un sentiment né de l'éveil des élites ottomanes aux idéaux nationaux propagés à partir des exemples européens. Les Turcs, constate Bernard Lewis, furent, parmi les peuples qui embrassèrent l'islam, ceux qui allèrent le plus loin dans la dissolution de leur identité ethnique dans la communauté islamique. «Jusqu'au début du $\mathrm{XIX}^{\mathrm{e}}$ siècle, les Turcs ottomans considérèrent la société dans laquelle ils vivaient comme le sommet de deux lignes de développement. La première commençait avec la mission de Muhammad, la montée de l'islam, et l'instauration du califat; la seconde avec l'avènement de la Maison d'Osman et de l'Empire Ottoman... Après la fidélité à l'égard de la communauté islamique venait la loyauté politique à l'égard de l'État islamique légal» ${ }^{7}$. Le régime kémaliste s'emploiera donc à fabriquer une identité nationale turque monolithique et jacobine par l'éducation, la propagande et la contrainte, et, principalement, en coupant la Turquie de son passé historique récent et de ses racines orientales.

La laïcité, pilier fondamental de l'idéologie kémaliste, apparaît comme le principe le plus marquant de l'identité de la République turque et donne la mesure de son originalité parmi les pays musulmans. Bien que Mustafa Kémal n'ait apparemment jamais conçu la laïcité comme un principe a-religieux -ce qu'elle n'est pas, la majorité de la population a quant à elle identifié la laïcité comme un principe athée, en fonction de sa formation culturelle selon laquelle on ne pouvait que se situer à l'intérieur d'un système socio-politique islamique ou en dehors. Loin, donc, de considérer la laïcité comme un élément garantissant la liberté de conscience des citoyens de la République, la population dans sa grande majorité l'a appréhendée comme une composante de l'idéologie dominante qui lui était imposée par le groupe détenteur de l'autorité politique.

Celui-ci, par ailleurs, a très tôt considéré que l'ouverture de la population turque à la modernité ne pouvait être assurée qu'à la condition d'extirper par voie autoritaire les références culturelles d'inspiration traditionnelle et islamique qui conditionnaient les approchent socioculturelles de la majorité de la population, et leur substituer de nouveaux systèmes de valeurs et de pensée en rapport avec les finalités séculières poursuivies par les promoteurs de la Turquie moderne. En ce sens, la laïcité a été imposée comme idéologie modernisatrice dans la société turque au travers du contrôle exercé par l'État sur l'espace public. La laïcisation de la Turquie a donc été la résultante d'un processus «d'ingénierie sociale plutôt que le résultat d'un processus de modernisation et de développement sociétal»8.

En fonction de cette approche des réalités du pays au début de ce siècle, les élites kémalistes ont conclu que le respect du principe démocratique de la souveraineté du peuple entraînait nécessairementl' acceptation de ses systèmes de valeurs d'inspiration islamique, et la renonciation 
au processus d'occidentalisation et de modernisation consécutive du pays. D'où le choix opéré, dans l'immédiat, par Atatürk et ses collaborateurs pour un régime autoritaire.

Il apparaît ainsi qu'à la création de la République turque, les notions de laïcité et de démocratie soient apparues comme essentiellement antinomiques. C'est pourquoi, vraisemblablement la démocratie n'a jamais figuré au côté des six flèches du Parti fondé par Atatürk ${ }^{9}$. Comme le souligne Nilüfer Göle, «cette dualité entre un État qui se pose comme seul acteur du progrès et l'autonomisation de la société dont les expressions sont considérées comme rétrogrades constituera le cercle vicieux (démocratisation/réislamisation) de la vie politique turque tout au long de la République. Comme nous le verrons, chaque phase de démocratisation a entraîné une nouvelle visibilité des valeurs islamiques; ce phénomène considéré comme une menace pour les principes laïques de la République, dès lors qu'il atteint un certain niveau, a justifié une intervention de l'establishment militaire et une altération plus ou moins brève de la vie démocratique ${ }^{10}$.

\section{L'AVĖNEMENT DUMULTIPARTISMEET LES PREMIËRES MANIFESTATIONSDU «RENOUVEAU RELIGIEUX» DE 1945 À 1960}

En novembre 1947, Ismet Inönü fait voter la réforme de la loi électorale, qui constitue une reconnaissance du multipartisme et instaure l'élection directe des députés au scrutin majoritaire.

L'essentiel du combat politique en Turquie va être monopolisé par l'affrontement entre le PRP, qui malgré ses efforts pour élargir son électorat, reste le parti de l'intelligentsia bureaucratique et des catégories sociales bénéficiaires de l'étatisme; le Parti Démocrate (PD), issu d'une scission du PRP, reçoit, quant à lui, le soutien de divers groupes sociaux qui se sentaient lésés par la gestion du PRP.

Dans la mesure où l'instauration du multipartisme permet une plus grande liberté d'expression aux diverses tendances représentées dans l'opinion publique, l'affirmation par la population de son identité religieuse gagne en visibilité. Toutefois, lorsque l'on parle de «renaissance religieuse» ou de «renouveau religieux», il convient de ne pas se tromper de débat : il ne s'agit pas d'une renaissance de la foi, qui, en Turquie, n'a jamais été en crise, mais bien, dans le contexte des années 1940 et 1950, d'un renouveau de l'expression publique de la religiosité. Dès la fin des années 1940 et tout au long des années 1950, les mosquées s'emplissent de pratiquants; les ouvrages, les quotidiens et les hebdomadaires populaires traitant de questions religieuses se multiplient dans les librairies et les points de vente publics. Les confréries (tarikat) et des hommes de religion de toutes tendances se manifestent en public pour remettre en cause les principes laïques de la République ${ }^{11}$.

Une grande partie de la bataille que se livrent les deux grandes formations politiques pour la conquête de l'électorat porta sur la revalorisation de la religion dans la société turque. Cette revalorisation fut activement encouragée par les États-Unis qui y voyaient un moyen d'enrayer la progression du communisme et de l'idéologie marxiste en Turquie. Dans ce contexte, Ismet Inönü fut, dès 1945, le promoteur d'une campagne exaltant les vertus de la religion musulmane. Contraint, par la suite, de prendre en compte les revendications d'un électorat séduit par les promesses du PD en matière de liberté d'expression religieuse il mit en chantier une série de réformes dans le domaine de l'expression publique de la religiosité.

Le 27 février 1947, le comité directeur du PRP autorise l'enseignement, à titre facultatif, par des maîtres agréés par le ministère de l'Education, de cours de religion dans les écoles 
publiques. Le 10 juin 1949, le gouvernement procède à la création d'une faculté de théologie annexée à la faculté de droit d'Ankara ${ }^{12}$.

Le 14 mai 1950, à l'issue des premières élections régulières organisées en Turquie, le PRP subit, avec $29 \%$ de voix et 69 sièges, une défaite retentissante face au PD qui remportait 53\% des suffrages et 420 sièges.

Fidèle à ses engagements électoraux, le PD généralisa les cours de religion dans l'enseignement public, rétablit l'usage de l'arabe dans l'appel à la prière, autorisa l'expression publique de la religiosité dans tous les domaines. Il fut toutefois rapidement débordé par la virulence de la réaction religieuse orchestrée par les confréries et nombre d'hommes de religion officiellement reconnus, qui réclamaient l'abrogation des structures institutionnelles laïques de l'État, le retour à la sharia et le rétablissement de structures étatiques islamiques, voire pour certains le retour à la monarchie.

En 1951, des manifestants appartenant à la confrérie de la Tidjaniya décapitent en plusieurs occasions des bustes d' Atatürk. Plusieurs manifestations dirigées contre les réformes kémalistes et contre Atatürk lui-même sont conduites tout au long des années 1950 par des adeptes de confréries religieuses, parmi lesquels les Nakchibendiler et le Nurcular apparaîtront comme les plus actives. Au sein du PD lui-même des députés, en session parlementaire, n'hésitèrent pas à proposer l'abrogation du code civil et le rétablissement de la sharia, l'inscription dans la Constitution de l'islam comme religion d'État, l'obligation pour les députés de prêter serment à l'Assemblée en posant la main sur le Coran ${ }^{13}$.

A l'égard de ses membres, coupables de vouloir remettre en question les structures laïques de la République, le PD réagit avec la plus extrême rigueur. Le Parti était, en effet, essentiellement, dans l'esprit de ses principaux dirigeants, la formation des milieux d'affaires et des entrepreneurs privés, dont le développement était lié à l'amarrage de la Turquie à l'économie de marché et à l'ancrage à l'Occident libéral d'où provenait la totalité de l'aide économique et de la protection contre la menace communiste. La réaction à l'égard des manifestations populaires hostiles aux institutions laïques de l'État fut nettement plus molle, malgré la promulgation d'une loi et de décrets destinés à protéger la mémoire d'Atatürk. C'est que la majorité de l'électorat du PD était d'origine rurale, conservatrice et fondamentalement hostile à la laïcisation de l'État; si le monde paysan avait majoritairement accordé ses suffrages au PD c'était non pas tellement en fonction de son programme économique libéral, mais surtout en raison de la partie de son programme relatif à la revalorisation de la religion dans l'espace public. Le PD était ainsi amené à pratiquer une politique d'équilibre entre deux impératifs difficilement conciliables : d'une part la nécessité d'approfondir l'intégration de la Turquie dans le monde occidental libéral; d'autre part le besoin de conforter son image de marque de défenseur de l'islam auprès de sa majorité électorale issue du monde rural.

Sur le plan politique, dans un État où la formation d'un parti fondé sur l'exploitation du sentiment religieux de la population à des fins politiques tombait sous le coup du droit pénal, les chefs de file des principales confréries et autres associations religieuses pratiquèrent, dès les années 1950 , l'entrisme au sein de formations politiques dont le succès était lié, en tout ou en grande partie, aux suffrages des populations conservatrices attachées aux valeurs de l'islam. C'est ainsi que d'une manière récurrente des partis étiquetés comme libéraux ou de «centredroit», défenseurs attitrés de la liberté d'expression religieuse dans l'espace public, devinrent le lieu où se trouvaient mêlés représentants du monde des affaires et du capitalisme turc le plus moderniste d'une part, et militants et élus membres de confréries d'autre part. 
L'équation démocratisation-réappropriation de l'espace public par des mouvements favorables à une réislamisation de la vie publique est pleinement vérifiée au cours de la période s'étendant de 1945 à 1960. A ce stade toutefois, les revendications religieuses reflètent les exigences d'un islam essentiellement populaire et témoignent d'une vision globalement passéiste. L'approche islamiste qui se développera au cours des deux décennies suivantes témoigne d'une évolution notable des courants de pensée des mouvances islamiques liée à l'évolution de la société turque elle-même.

Cette évolution est réalisée parallèlement aux mouvements de populations qui ont contribué à changer significativement la nature du peuplement de la capitale et des grandes métropoles. Dans le but de se ménager une clientèle électorale qui lui soit favorable dans les grands centres urbains, le PD y attira les paysans en leur offrant des emplois rémunérateurs grâce à la mise en chantier de grands travaux. La politique agraire du PD en favorisant la mécanisation de l'agriculture eut pour effet de raréfier l'emploi dans le secteur agricole et favorisa un exode rural qui n'a cessé, depuis, d'engorger les grandes métropoles. Les populations paysannes qui s'y installent apportent avec eux leurs coutumes, leurs manières de vivre et leurs croyances religieuses. La physionomie de la population urbaine en Turquie change radicalement depuis les années 1950.

L'industrialisation, la modernisation économique, l'urbanisation et l'exode rural ont engendré des phénomènes de fragmentation et de segmentation de la société ainsi qu'un relâchement des liens familiaux et des relations sociales. L'ensemble de ces facteurs a été décisif pour le développement des mouvements religieux. Les paysans ayant immigré dans les villes pour des raisons économiques, coupés de leurs racines, isolés dans un milieu ressenti comme peu accueillant, affectés par un recul général de la famille comme élément traditionnel d'intégration sociale, ont trouvé au travers de l'appartenance à des mouvements religieux un moyen de reconstituer un tissu de relations sociales sécurisant. La religion, pour reprendre la vision durkheimienne, par son autorité morale et ses aspects rituels de groupe constitue une force de cohésion de la collectivité; elle apparait alors comme un facteur de restauration de la solidarité de groupe. En ce sens, Metin Heper parle, à propos de ce que l'on a désigné comme une renaissance de la religion en Turquie, de l'expansion d'une religion séculière plutôt que d'une intensification de la foi en tant que croyance irrationnelle ${ }^{14}$.

\section{LADEUXIĖMERÉPUBLIQUE : DÉMOCRATISATIONDE LA VIE PUBLIQUE ET ÉVOLUTIONDES COURANTS ISLAMIQUES}

Le 27 mai 1960 l'armée excipant de son rôle de gardienne du respect des principes kémalistes, décida de renverser le gouvernement dirigé par Adnan Mendérès, jugé coupable d'avoir plongé le pays dans une crise économique aiguë, d'avoir paralysé la vie parlementaire et de s'être montré peu enclin à réprimer efficacement les mouvements religieux qui remettaient en question les institutions laïques de la République. La répression énergique des activités antiläques figura parmi les premières mesures prises par les militaires au pouvoir.

La junte militaire installée aux commandes de l'État sous la direction du général Cemal Gürsel créa, avant de remettre le pouvoir aux civils, un Conseil National de Sécurité où siègent le Président de la République, le Premier ministre, le chef d'état-major et les commandants des trois armées. Ce Conseil devint, et reste à ce jour, l'une des institutions essentielles de l'État et le garant de la préservation de l'héritage idéologique kémaliste. 
Dans le même esprit, le Comité d'Union Nationale, dominé par des officiers d'orientation majoritairement réformiste, fit promulguer la Constitution la plus libérale qu'ait connue la Turquie. Le droit de grève fut ainsi reconnu pour la première fois depuis 1925; la liberté d'opinion, de presse, d'expression et d'association fut garantie.

La libéralisation de la vie politique initiée par la junte militaire en 1960-1961 eut une influence significative sur l'évolution de la culture politique de la population turque, en dépit des entraves que la guerre froide et l'anticommunisme primaire qu'elle avait suscité, avaient mises à la libre expression d'opinions politiques en contradiction avec les credos officiels. La création en 1961 d'un Parti Ouvrier de Turquie, toléré malgré la présence à sa tête de personnalités marxisantes bien connues, le développement d'une activité syndicale dynamique, la publication, la diffusion et la traduction d'ouvrages marxistes exercèrent une influence nonnégligeable sur une partie de l'intelligentsia et du monde étudiant. Parallèlement l'approfondissement d'une réflexion tendant à promouvoirl'intégration du concept de modernité dans la pensée religieuse, et la possibilité offerte à celle-ci d'être discutée dans l'espace public, est une autre manifestation des conséquences de l'ouverture démocratique de la société turque. Enfin, l'intégration croissante de la Turquie dans l'économie libérale à l'échelon mondial eut aussi pour effet d'influer sur la dimension libérale de la pensée politique de nombre d'acteurs du monde économique et de leurs relais politiques. Bref, il n'est pas exagéré d'affirmer que la réflexion politique n'a cessé de s'affiner, des années 1960 aux années 1990, au niveau de la population prise dans sa globalité, malgré les restrictions imposées àl'exercice de la démocratie par les coups d'État militaires de 1970 et 1980.

L'importance des années 1960 et 1970 dans l'évolution de la vie politique apparaît tout particulièrement dans la mesure où elles donnèrent naissance à des formations politiques qui témoignent de la diversification des options politiques et continuent, moyennant, pour certains, des changements d'appellation, à marquer la vie politique de nos jours.

En 1961, fut créé le Parti Ouvrier de Turquie déjà cité, seule des quatre formations mentionnées ici à disparaître de la scène politique à la suite du coup d'État de 1980.

Autre formation politique créée dans les années 1960 le Parti du Mouvement National, fondé en 1969 par l'ex colonel Alparslan Türkes, présente un programme ultranationaliste et pan-turkiste prônant l'instauration d'un État fort pourvu de structures politiques, économiques et sociales de type fasciste. Pour appuyer ses revendications politiques ce parti mettra sur pied des groupes paramilitaires qui se désigneront sous l'étiquette de «loups gris», et dont l'action violente, principalement contre les mouvements de gauche, dans la rue et dans les universités, constituera un facteur de déstabilisation de l'État dans les années 1970.

En 1972, sous la houlette de Bülent Ecevit le PRP se mue en parti social-démocrate dans la tradition des partis sociaux-démocrates européens.

La même année est créé, le Parti du Salut National dirigé par Necmettin Erbakan, ingénieur de formation, qui préconise le développement d'une économie libérale, dont les effets sociaux seraient conditionnés par l'application des principes solidaristes et fraternels qui constituent l'essence d'une société islamique. Pour assurer un retour à une société islamique, dont les valeurs essentielles seraient préservées notamment sur le plan éthique, ce parti propose une restructuration institutionnelle qui ferait de la Turquie un État musulman moderne. Sur le plan de sa conception économique, le programme du PSN rappelle furieusement celui du mouvement Nurcu, qui fut d'ailleurs l'un des soutiens de départ à la constitution du Parti islamiste. Le PSN, n'est toutefois pas, au contraire du mouvement Nurcu, le lieu où se développe une pensée 
islamiste sophistiquée. Erbakan et ses proches collaborateurs ne sont pas des penseurs islamiques et leur connaissance de l'islam est relativement rudimentaire. Le succès du parti est essentiellement dû au fait qu'Erbakan a fort bien saisi l'état d'esprit des populations rurales migrantes qui, dans les villes, ont été confrontées aux comportements méprisants des citadins; en outre, coupés de leurs sources traditionnelles de soutien et d'identité, ils ont dû faire face, en isolés, à d'importantes difficultés économiques dues en grande partie à une inflation à trois chiffres depuis les années 1970. Face à ces déracinés, en butte à des difficultés matérielles qui résultent d'une évolution économique mal maîtrisée, le parti d'Erbakan a su trouver les références mobilisatrices en termes religieux identifiables par cette population, en se présentant à elle comme le parti de «l'ordre juste» (adil düzen) face aux tenants d'une occidentalisation accusée de générer les maux dont souffrent les couches défavorisées de la population, lesquels représentent «l'ordre des injustes» ${ }^{15}$.

La diversification des prises de position politiques, après 1960, s'inscrivait dans un contexte de mutations sociales accélérées. A cette époque, en effet, on assiste à une régression de l'importance politique des groupes ruraux tandis que les professions libérales, les techniciens et les milieux d'affaires acquièrent un poids politique sans cesse croissant dans une société où la différenciation et la spécialisation des groupes sociaux s'accentuent au sein du corps social. Corollaire de cette évolution, les oppositions d'intérêts entre ces divers groupes s'accentuent. Les milieux industriels, en pleine expansion, les groupes de travailleurs, les détenteurs de capitaux, certains secteurs de l'intelligentsia bureaucratique se transforment progressivement en groupes de pression. Cette transformation de groupes sociaux en groupes de pression va remplacer l'opposition traditionnelle entre groupes économiques et groupes bureaucratiques et étatistes caractéristique de la première république. La force dynamique essentielle de l'action politique après 1960 est l'intérêt individuel focalisé sur la recherche d'une amélioration des conditions de vie matérielles. C'est probablement la principale raison qui explique que l'on n'ait pas assisté à la formation de parti de classe. Ce qui explique la faible audience du POT qui fut essentiellement un parti d'intellectuels ${ }^{16}$.

Il n'en reste pas moins que l'on voit se dessiner à partir des années 1960 une bataille idéologique entre l'avant-garde du groupe étatiste qui finit par embrasser la social démocratie et le groupe des milieux d'affaire et de l'entreprise industrielle qui épouse les thèses libérales. Mais ces courants idéologiques ne se rattachent pas, contrairement à ce qui s'est passé dans le monde occidental, à une école de pensée mais à des conditions matérielles spécifiques à la Turquie. L'apport des retombées du coup d'État, de 1960 est d'avoir ouvert, aux diverses forces sociales en formation, les voies d'un ordre politique potentiellement représentatif, et d'une confrontation des forces politiques déjà comparable à celle qui prévaut dans les démocraties libérales occidentales ${ }^{17}$.

Cette évolution manifeste au niveau de la réflexion politique s'accompagne d'un développement de la pensée politico-religieuse qui épouse elle-même l'évolution de la société turque.

Dans le courant des années 1950, 1960 et 1970, les groupes issus de la périphérie, immigrés dans les centres urbains, et tout particulièrement leurs descendances, ont eu accès à l'enseignement laïque et ont connu les effets d'une certaine mobilité sociale. Paradoxe apparent, plus les groupes issus de la périphérie ont eu accès à la vie urbaine, à une éducation libérale et à de nouveaux modes d'expression politique, plus ils apparaissent disposés à rechercher des sources de référence islamiques pour redéfinir leur identité et leur place dans la société globale. 
Les mouvements islamistes, qui ont suivi une nouvelle évolution à partir des années 1960 , ont essayé de répondre aux aspirations de ces nouveaux groupes et de les aider à se réconcilier avec la modernité. Aux musulmans de la périphérie, ces mêmes mouvements ont essayé de procurer une sorte de guidance pour définir une ligne de conduite adaptée à leur vie quotidienne et leur fournir de nouvelles formes d'expression politique ${ }^{18}$.

Sensible au niveau des masses, l'influence du courant islamiste se fait aussi ressentir au niveau de l'intelligentsia. Encore convient-il de préciser la nature de cette «islamisation» d'une partie de l'intelligentsia turque.

Les intellectuels turcs, remarque Metin Heper, formé dans le contexte de la République kémaliste, ont évolué dans un cadre où la vie de la cité a été désacralisée et où s'est opérée une sécularisation normative progressive. Cette évolution a été précipitée par les effets conjugués de l'industrialisation de l'urbanisation et de l'expansion des médias dans les années 1950 et 1960. Au cours de ces périodes successives, les intellectuels turcs, y compris ceux pour lesquels l'islam est redevenu un élément de leur identité, ont fait non seulement l'expérience de la «sécularisation normative comme processus de désacralisation» mais aussi de la «sécularisation cognitive comme segmentation». Ainsi selon Metin Heper - dont nous rejoignons totalement l'évaluation sur ce point - les intellectuels turcs musulmans du début des années 1980, ne se différencient-ils pas fondamentalement des élites croyantes occidentales dans leur vision du monde profane et de la place qu'y occupe la religion ${ }^{19}$.

La modernisation de la pensée politico-religieuse, en Turquie, doit beaucoup aux développements théoriques du mouvement Nurcu, qui s'est rapidement trouvé en prise avec les nouveaux groupes professionnels émergents composés de diplômés universitaires issus en grande partie de milieux populaires.

La deuxième moitié des années 1960 est marquée par la montée spectaculaire du mouvement Nurcu, influant dans les provinces, mais aussi infiltré dans la bureaucratie et les milieux universitaires. Militants actifs, les Nurcular animèrent à plusieurs reprises des manifestations dirigées contre des réunions du POT au nom de la lutte contre le communisme ${ }^{20}$.

L'exégèse des écrits de Bediüzzzaman Said Nursi (fondateur du mouvement Nurcu, 1873 1960), par ses disciples les plus en vue, donne du mouvement une vision moderniste : l'ouverture à l'innovation, aux sciences et à la recherche scientifique devient ainsi l'une des vertus cardinales du bon musulman. Selon ces mêmes exégètes, le Nurcu type est un citoyen respectueux de la «démocratie républicaine et pluraliste» pour lequel la laïcité de l'État est acceptable à la condition que l'État renonce à exercer son contrôle sur la vie spirituelle des citoyens. Cette acceptation de la laïcité de l'État par le mouvement apparaît contradictoire avec sa volonté affirmée, par ailleurs, de développer une action visant à établir les bases politique, sociale et économique de l'État selon des critères islamiques, c'est-à-dire, in fine, d'établir un contrôle de l'islam sur l'État.

Sur le plan de l'organisation économique d'une société islamique selon sa conception, le mouvement Nurcu condamne comme également contraire à l'esprit de l'islam le capitalisme et le communisme, qui développent tous deux une vision matérialiste du monde. En effet, le premier est fondé sur l'usure et l'appât du gain qui conduisent inévitablement à des déséquilibres sociaux majeurs inacceptables au regard de l'islam; le second prive tout citoyen de toute forme de liberté, y compris celle de pouvoir jouir du fruit de son travail. La troisième voie préconisée par le mouvement Nurcu, conforme à l'esprit de l'islam, est une sorte de libéralisme tempéré 
par la mise en place des garde-fous sociaux que constitue le respect des obligations et interdits prescrits par l'islam. En d'autre mots, ce système économique d'inspiration islamique est fondé, d'une part, sur la libre entreprise et l'économie de marché, d'autre part sur l'obligation pour l'État d'intervenir en matière de planification du développement économique, de création d'un système bancaire islamique efficace et de dépenses d'infrastructures profitables à l'ensemble de la société. Le souci d'équilibre socio-économique dont témoigne ce programme s'inscrit dans le respect des préceptes de la révélation, qui, seul, assure le bonheur des hommes et le règne de l'équitée ${ }^{21}$.

La confrérie de la Nakchibendiya est, avec le mouvement Nurcu, l'organisation islamiste qui rassemble le plus grand nombre d'adhérents. Plus conservatrice sur le plan théologique, la confrérie la rejoint toutefois sur l'importance accordée aux sciences, à la technologie et à l'enseignement comme facteurs essentiels du développement des sociétés. Comme son concurrent Nurcu, elle attire une clientèle d'ingénieurs, de techniciens et d'intellectuels auxquels elle offre un soutien au niveau de la promotion professionnelle, grâce à ses réseaux de solidarité et d'influence. Comme les Nurcular, les Nakchibendiler, se montrent virulemment anticommunistes et ont pris, durant l'époque de la guerre froide, une part active dans les manifestations souvent violentes dirigées contre les formations de gauche.

En 1970, le mouvement Nurcu et la Nakchibendiya furent parmi les principaux soutiens de la formation d'un parti islamiste, le Parti de l'Ordre National (Millî Nizam Partisi), fondé par Necmettin Erbakan ${ }^{22}$, qui fut interdit en 1971, pour avoir exploité les sentiments religieux de la population à des fins politiques. Il fut remplacé peu après par le Parti du Salut National (Millî Selamet Partisi) dont le programme insistait sur la nécessité de créer un «capitalisme national» et opérer avec les pays musulmans un rapprochement qui devrait déboucher sur la création d'un marché commun des pays musulmans afin d'échapper à la tutelle oppressive et perverse du capitalisme occidental. Parmi les ennemis les plus résolus de la Turquie il citait l'Occident foncièrement antimusulman, le sionisme, la franc-maçonnerie et les papistes.

Depuis les élections de 1973 et jusqu'au coup d’État de 1980, le PSN participa sans interruption à tous les gouvernements de coalition qui se succédèrent durant cette période. Il en profitera pour placer des hommes à lui aux postes clés dans plusieurs ministères. Ces derniers exerceront notamment une influence considérable dans le domaine de l'éducation nationale et notamment au niveau de l'enseignement religieux. Les écoles publiques accordèrent une place de plus en plus importante à l'enseignement de la religion. De 1973 à 1977, le nombre d'écoles de prédicateurs et de directeurs de prière est passé de 143 à 320 , le nombre d'étudiants de plus ou moins 36.000 à 111.741 et le nombre d'enseignants de 1.564 à 3.852 . en outre les manuels scolaires accorderont une place prépondérante à la célébration de la grandeur de l'empire ottoman et présenteront l'islam comme l'une des bases essentielles de la civilisation et de la culture turques ${ }^{23}$.

Depuis l'établissement du Millî Nizam Partisi, 1970, l'islamisme turc a été incorporé dans le système politique et légitimé par le système parlementaire. Ce processus de légitimation a été interrompu par les coups d'État militaires de 1970 et 1980, qui ont chaque fois interdit le parti islamiste. Néanmoins, celui-ci a été reconstitué chaque fois sous l'appellation de Parti du Salut National de 1972 à 1980, et sous celle de Parti de la Prospérité de 1983 à 1997, et a participé à plusieurs gouvernements de coalition ${ }^{24}$.

Dans la mesure, toutefois, où le sens politique de l'ensemble du corps social a évolué de manière significative depuis 1960, l'électeur a progressivement appris à se servir de son 
bulletin de vote pour sanctionner l'action concrète des formations politiques en lices sur le plan socio-économique et, moins qu' auparavant, pour soutenir un parti en fonction de son engagement religieux. Il est ainsi significatif de constater qu'à l'issue des élections de 1973, le PSN avait remporté 48 sièges à l'Assemblée nationale, alors qu'il n'engrangeait plus que 20 sièges en 1977.

Ce constat ne doit toutefois pas nous amener à conclure qu'à chaque baisse des résultats électoraux du PSN correspond une régression de l'islamisme. Le PSN, et le RP à sa suite, n'ont jamais été les seuls représentants de l'islamisme au niveau politique. Des mouvements comme celui des Nurcular, ou des confréries comme la Nakchibendiya, qui ont à certains moments retiré leur confiance au parti de Necmettin Erabakan, ont continué à infiltrer les partis de centre droit; comme le Parti de la Justice et; après le coup d'État de 1980, le Parti de la Mère Patrie, et à jouer par ce biais un rôle politique non négligeable. En outre, le Parti du Mouvement National, d'inspiration fasciste, dans le souci d'élargir l'éventail de son électorat, avait lui aussi intégré le paramètre islamique dans son programme idéologique, et liait étroitement identité turque et identité musulmane. Le slogan du PMN devint ainsi : se ressentir comme musulman pour se sentir totalement turc.

Quelle que soit donc l'aspect sous lequel il se manifeste, l'activisme islamique est devenu depuis les années 1960 une composante incontournable de la scène politique turque. Sous toutes les formes que lui impriment ses diverses composantes, l'islamisme apparaît fondamentalement comme un mouvement de réappropriation des valeurs et de l'identité musulmane comme base pour un projet politique et social alternatif ${ }^{25}$.

\section{LE COUP D'ÉTAT DE 1980 ET LES DÉVELOPPEMENTS DE L'ISLAMISME SOUS LA TROISIÈME RÉPUBLIQUE}

Le coup d'État de 1980 permit à l'armée de réaffirmer une fois de plus son attachement à la préservation du caractère laïque de l'État et à celle des principes essentiels de l'idéologie kémaliste. La religion, à la condition de ne pas servir de prétexte à la remise en question de la nature de l'État turc, se voyait reconnaître tous ses acquis en matière de visibilité dans l'espace public. Plus même l'establishment militaire et nombre de membres de l'élite représentant l'État commencent à intégrer dans leur discours des références à l'identité musulmane des Turcs.

Cette reconnaissance d'une certaine identité musulmane turque est semble-t-il dictée par deux impératifs. Tout d'abord dans le contexte de la guerre froide, l'islam continue, plus que jamais à être considéré comme le barrage le plus mobilisateur contre une possible expansion de l'idéologie marxiste. Il apparaît ensuite de la manière la plus évidente, que tout retour à la démocratie, même dans une mesure limitée, ramènerait inévitablement un parti islamiste, sous quelle que appellation que ce soit, sur la scène politique. Éradiquer la présence d'islamistes de la vie politique turque paraît un leurre. Il ne fait, en effet, aucun doute, pour quel que acteur politique que ce soit, que certains ordres et mouvements religieux ont investi depuis de longues années des partis politiques parmi les plus importants, de sorte qu'interdire la création d'un parti islamiste n'aurait aucune incidence réelle sur l'existence même de mouvements islamistes, sinon à accroître l'audience des éléments anti-laïques les plus radicaux. Par ailleurs aucun groupe ayant une influence ou un pouvoir politique quelconque, y compris l'establishment militaire et une majorité d'islamistes, ne marque une préférence pour un régime autoritaire plutôt que pour la démocratie. Plus que jamais les années qui ont suivi le coup d'État de 1980, ont prouvé que «l'islam a été intégré dans la démocratie turque de diverses façons, tandis que la laïcité constitutionnelle et légale a été préservée» ${ }^{26}$. Cette intégration a, il est vrai, été 
largement favorisée par l'évolution des principaux portes parole de l'islamisme. et par l'évolution d'une partie des élites qui se sont attachées à redéfinir l'identité officielle turque au travers d'une réflexion historique dont l'aboutissement est la formulation d'une «synthèse turco-islamique». Cette démarche intellectuelle, en vogue dans les années 1980, a essentiellement visé à apporter une nouvelle dimension à l'image officielle de l'identité turque telle qu'elle avait été forgée à l'époque kémaliste : sans renier les brillantes origines centro-asiatiques exaltées par l'historiographie du régime kémaliste, l'accent sera également mis sur la civilisation prestigieuse édifiée par l'Empire ottoman et sur le rôle primordial joué par les Turcs dans la défense et l'expansion de l'islam. Au terme de sa réflexion, la «synthèse turco-islamique» a ambitionné de marquer l'aboutissement d'une longue quête d'identité poursuivie par le peuple turc depuis la mise en place de réformes politico-culturelles kémalistes.

A ces facteurs internes de réévaluation de la place de l'islam dans la formation de l'identité turque, se sont ajoutés les effets du rapprochement opéré par la Turquie avec le monde arabe et musulman à partir de la deuxième moitié des années 1970. En fait, des relations suivies, quoi que à une moindre échelle que dans les années 1970 et 1980, entre activistes musulmans de Turquie et penseurs islamiques d'autres pays musulmans, remontent à 1950, lorsque l'arrivée au pouvoir du Parti Démocrate amène la Turquie à envoyer des étudiants en théologie à $\mathrm{Al}$ Azhar et à la Faculté de théologie de Bagdad. Ce sont ces anciens étudiants qui contribueront, à la fin des années 1960, à la diffusion des écrits, préalablement traduits en turc, de Sayyid Qutb et Abu 'l-Ala al Mawdûdî, puis à ceux de Hassan al-Banna, fondateur du mouvement des Frères musulmans. Par la suite un nombre croissant d'étudiants en théologie seront envoyés en Arabie Saoudite, où certains d'entre eux suivront les cours de l'un des fils de Sayyid Qutb, qui entretient des relations suivies avec les islamistes turcs. De son côté le mouvement Nurcu tente de faire connaître ses idées en Égypte par le canal de madrasas qu'il y a fondées ${ }^{27}$.

Les échanges entre islamistes arabes et turcs allaient encore être amplifiés en raison de l'approfondissement et l'intensification des relations politico-économiques entre la Turquie et le monde musulman. Pour asseoir sa présence dans le monde musulman, la Turquie allait participer aux activités des organisations islamiques comme l'Organisation de la Conférence islamique. L'ouverture des marchés moyen-orientaux, notamment ceux de l'Arabie Saoudite, aux produits et aux entreprises turcs, allait, par contre coup, ouvrir la Turquie au militantisme islamique de certains États musulmans. L'Arabie Saoudite, principalement, en raison du débouché important qu'elle représentait pourl'économie turque, et profitant d'une conjoncture favorable sous le gouvernement, puis la présidence de Turgut Özal, oeuvra activement à la réislamisation de la société turque, subsidia abondamment diverses associations islamistes turques ainsi que la construction de mosquées dans le pays.

Toutefois, en dépit de sa générosité l'Arabie Saoudite n'apparut à aucun moment comme un modèle de référence pour la Turquie, où même les islamistes avaient intégré des modes de vie, des comportements et des visions du monde fort éloignés de ceux des Saoudiens. Entre les mouvements turcs et les mouvements orientaux existent des divergences idéologiques et des différences de culture politique considérables. Toutefois, sur les plans de la réflexion théologique et théorique, ainsi que dans le domaine de l'argumentation, l'influence des Frères musulmans reste importante. Cette importance accordée à la relation entre islamistes turcs et Frères musulmans trouve son expression la plus significative dans le fait que ces derniers sont représentés dans tous les congrès annuels du Parti de la Prospérité (PP), où l'on retrouve notamment Sayf Allah al-Banna, fils de Hassan al Banna, marié à une turque ${ }^{28}$. 
L'apparition de nouveaux maîtres à penser turcs et de nouveaux groupes sociaux dominants islamistes dans les années 1980 , a contribué dans une large mesure à donner au mouvement islamiste une dimension et une profondeur théoriques, comparables à celle de ses équivalents orientaux, et à lui conférer une vision plus ouverte sur la modernité.

Depuis une vingtaine d'années on assiste à l'émergence d'intellectuels islamistes comme Ali Bulaç, Ismet Özel, Rasim Özdemir et Abdurrahman Dilipak. Comme l'intelligentsia laïque, ces nouveaux penseurs de l'islam, dispensateurs de symboles et de valeurs, ont suivi un enseignement laïque, maîtrisent une ou plusieurs langues étrangères, connaissent les littératures occidentales, s'intéressent à la production philosophique de l'Occident, et suivent l'évolution de sa pensée politique. Fort influents dans les milieux universitaires, ils se trouvent fréquemment engagés dans des débats publics avec leurs homologues laïques avec lesquels ils partagent une formation scolaire et un bagage conceptuel communs. Faisant référence au modèle de société musulmane originelle, inauguré par le Prophète et les quatre premiers khalifes, qui selon ces intellectuels islamistes n'est «ni théocratique (sic) ni totalitaire», dans la mesure où «c'est la communauté et non le leadership politique ni le clergé (sic)» qui était l'arbitre en dernière instance de la gestion de la chose publique. D'une manière globale, leur action vise à susciter dans la population une prise de conscience islamique et à promouvoir l'avènement d'une communauté musulmane pluraliste, non à oeuvrer à l'instauration d'un État islamique stricto $\mathrm{sensu}^{29}$.

Tout aussi importante pour l'avenir du courant islamiste est l'apparition sur la scène politique d'intellectuelles islamistes féminines, comme Cihan Aktas, Sibel Eraslan, Hamde Toros, fort actives dans la propagation de l'idéologie du mouvement.

Dans toutes les sociétés musulmanes l'une des manifestations de la visibilité de l'islam est le voile des femmes. Le voile symbolise l'islamisation du mode de vie... Il rappelle la définition traditionnelle de la condition féminine basée sur la modestie et la vertu, la ségrégation des sexes et l'interdiction pour les femmes de participer à la vie publique. Le voile aujourd'hui symbolise la participation politique de la femme et la réappropriation de son identité islamique. Elle assume une carrière professionnelle. Elle acquiert par là une visibilité dans l'espace public. Par conséquent l'image qu'elle offre n'est pas celle, effacée, de la tradition islamique. Mais le fait qu'elles ont une visibilité dans l'espace public, qu'elles exercent des professions où elles sont appelées à côtoyer des hommes et qu'elles ont des ambitions professionnelles à créer des tensions entre leur rôle de militante islamiste et leur rôle comme femme membre d'une contre élite dans une société sécularisée. D'où leurs relations difficiles non seulement avec les femmes laïques mais aussi avec les hommes islamistes ${ }^{30}$.

La transformation des cadres de l'islamisme que nous venons d'évoquer se manifeste d'une manière significative au niveau des confréries, comme la Nakchibendiya, et de certains mouvements religieux activistes, comme le courant Nurcu, dont l'action dans l'espace public n'a cessé de s'affirmer après le retour au pouvoir civil qui a suivi le coup d'État de 1980.

Dans l'ensemble, ces formations apparaissent de mieux en mieux intégrées dans l'État démocratique laïque. Outre le soutien accordé au nouveau parti islamiste, le Parti de la Prospérité, fondé en juillet 1983, poursuivant leur stratégie d'infiltration des partis de centredroit créés après le coup d'État de 1980, on retrouve nombre de leurs représentants dans le Parti de la Juste Voie (Dogru Yol Partisi), et tout particulièrement dans le Parti de la Mère Patrie, dont le président Turgut Özal qui fut lui-même un membre de la Nakchibendiya. Fait caractéristique des années 1980 et 1990, la Nakchibendiya, comme le mouvement Nurcu ont systématiquement 
retiré leur appui aux formations ou aux hommes politiques jugés trop ostensiblement menaçants pour le système politique en place, dans la mesure où une remise en question radicale de l'héritage laïque kémaliste pourrait provoquer une intervention de l'armée dont ils seraient les premiers à faire les frais; en outre ils estimaient non sans raison que le système de démocratie représentative, soutenu par les nouveaux intellectuels islamistes, ne leur avait pas trop mal réussi. La position politique de la majorité des membres du mouvement Nurcu est ainsi parfaitement explicitée par le fondateur du groupe des Fethüllahcilar, issu du mouvement lorsqu'il déclare dans une interview que le groupe «ne soutient pas n'importe quel gouvernement. Mais si quelqu'un est élu à une charge politique, nous nous conduirons à son égard comme les populations dans les pays occidentaux développés... L'État est très important. Son absence crée l'anarchie. J'ai toujours, dit-il encore, pressé les citoyens qui venaient prier à voter en leur disant que c'était le devoir de tout citoyen ${ }^{31}$.

Non sans pertinence, Nilüfer Göle, attribue aussi à l'investissement du champ politique, tout particulièrement islamiste, par l'ingénieur une importance non négligeable dans l'évolution de la vision du monde des courants islamistes modernes. Le prestige de la fonction d'ingénieur vient de ce qu'ils étaient considérés depuis les années 1950 comme l'élément moteur par excellence du développement. Les ingénieurs représentent actuellement une part non négligeable de la représentation parlementaire et figurent en nombre dans les rangs du Parti de la Prospérité

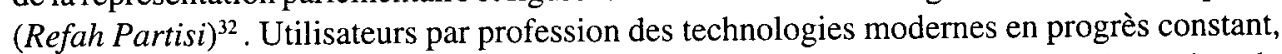
ils sont, de surcroît, en contact permanent avec le monde occidental d'où leur vient la technologie. Imbriqués dans le courant d'échanges économiques international qui fait de l'Occident industrialisé un partenaire obligé, ils peuvent difficilement s'engager dans une voie politique qui prônerait la rupture avec ce dernier. D'où le rôle joué par des ingénieurs entrés en politique dans la mise en cuvre d'une attitude de compromis entre l'affirmation d'une identité culturelle turco-islamique et l'ancrage à l'Occident partenaire économique obligé et source de progrès technologique garant de la prospérité de la Turquie. Cette attitude, qui s'inscrit parfaitement dans le courant de la synthèse turco-islamique, fut celle d'ingénieurs comme : Süleyman Demirel, président de deux partis de centre-droit, avant de devenir président de la République; Turgut Özal, président du Parti de la Mère-Patrie (Anavatan Partisi-ANAP), puis Président de la République. Necmettin Erbakan, autre ingénieur à avoir été président de parti, avant de devenir brièvement Premier ministre, représente un cas particulier dans la mesure où, contrairement aux deux autres il va prôner le distanciement de la Turquie de l'Occident et la formation d'un marché commun islamique comme solution alternative à la dépendance vis à vis de l'Europe et des État-Unis.

Parti représentant l'islamisme par excellence le Parti de la Prospérité, héritier et prolongement du Parti du Salut National, interdit à la suite du coup d'État de 1980, va connaître une évolution comparable aux autres mouvements islamistes en raison principalement du renouvellement progressif de ses cadres et de l'évolution du corps électoral en général.

Depuis sa création en 1983, le Parti de la Prospérité a repris les activités sociales et caritatives qui avait fait du Parti du Salut National, le parti favori d'une importante partie des nécessiteux. En outre, il pouvait compter sur l'appui de chefs d'entreprises islamistes qui rejoignaient ses rangs en nombre croissant, et se regroupaient dans des associations professionnelles affichant le cachet islamiste. Dans les années 1980/90, il apparaîtra, plus que jamais, que son audience croissante est due à sa présence sur le terrain économique et social plus qu'à sa qualité de parti islamiste. Il apparaît ainsi dans un sondage réalisé en 1994, que seul un tiers de son électorat a voté pour le Parti de la Prospérité (PP) parce qu'il était un parti 
islamiste ${ }^{33}$. De même il convient de relativiser la percée opérée par le PP, devenu le premier parti de Turquie aux termes des élections législatives de 1995. En effet, les formations de centre-droit et de centre-gauche auraient inévitablement occupé les première et deuxième places, si elles n'avaient été divisées par d'interminables querelles de personnes, et si de surcroît les chefs de file des partis de centre-droit n'avaient été compromis dans des affaires de corruption.

Face à des adversaires divisés et incapables de présenter des programmes attractifs, principalement pour les catégories de populations victimes des retombées du modèle de développement économique et de gestion sociale choisi par le pouvoir en place, le parti d'Erbakan avait mobilisé tout l'éventail des instruments de propagande dont il s'était doté. Il put ainsi compter sur le soutien d'un réseau complexe d'associations d'entreprises industrielles islamiques, de chambres de commerce et d'industrie, de syndicats, de groupements de jeunes, d'associations féminines, de quelque cinquante maisons d'édition, de quarante-cinq stationsradio, de dix-neuf chaînes de télévision et de centaines de producteurs de vidéos et de cassettes $^{34}$.

Outre, les groupes de pression dont il s'était doté, le PP s'était aussi efforcé d'asseoir sa popularité dans les quelque trois cent vingt-sept municipalités qu'il avait conquises à l'issue des élections municipales de 1994, grâce à la distribution de charbon, de nourriture et de vêtements aux plus nécessiteux de ses administrés, auxquels il assurait aussi des soins de santé gratuits dans les hôpitaux publics; cerise sur le gâteau, des cérémonies de circoncision sont payées pour les enfants des familles les moins favorisées. En outre, en 1997, selon le rapport d'observateurs neutres, dans les municipalités dirigées par des maires du PP, qui sont généralement considérés honnêtes et efficaces en matière de services municipaux, les ordures sont ramassées régulièrement, les services sociaux se sont améliorés, des travaux d'utilité publique ont été entrepris, des terrains de jeu pour enfants se sont développés ainsi que les espaces publics plantés d'arbres ${ }^{35}$.

Ces constats positifs doivent toutefois être nuancés en raison de l'image moins idyllique que donnent les récentes enquêtes menées dans les municipalités dirigées par des élus du PP.

Ainsi, des contrôles exercés dans les municipalités dirigées par le PP laissent apparaître des non-paiements de sommes importantes relatives à des primes de sécurité sociale dues à l'administration de la sécurité sociale. En outre, le ministère de l'intérieur du gouvernement ayant succédé à la coalition Parti de la Juste Voie (PJV)-PP, signalait que les municipalités du Grand Istanbul ont perdu 80.000.000 de dollars entre 1994 et 1997; il est apparu, de plus, que les services municipaux qui constituaient la vitrine de la gestion sociale de PP étaient largement financés par des emprunts extérieurs non payés. Ces dettes d'un montant de quelque cinq milliards de dollars ont dû être épongées par le trésor public ${ }^{36}$.

Enfin, en contradiction avec les professions de foi libérales des élites islamistes déjà citées, des élus RP dans les municipalités qu'ils contrôlaient ont tenté d'imposer, à leurs administrés, dans des domaines touchant à la vie privée comme dans l'espace public, des mesures de morale islamique visant à remettre en question des styles de vie considérés comme représentatifs de l'influence occidentale. C'est donc dans le sens d'une réislamisation des comportements quotidiens et des valeurs qu'il faut considérer l'interdiction de vendre de l'alcool dans les kiosques municipaux de Gaziantep et dans les restaurants de Kayseri, la création de piscines et de plages séparées pour hommes et pour femmes dans un hôtel cinq étoiles à Dydime, le retrait de statues de certains lieux publics à Ankara et Istanbul, le retrait sur ordre du maire d'une 
localité de la province d'Afyon d'une statue de taureau symbole de l'élevage, principale activité de l'endroit, sous prétexte qu'elle représentait une idole ${ }^{37}$.

Ces initiatives, qui témoignent d'un militantisme islamique rabique, contrastent avec les positions résolument ouvertes adoptées au quatrième congrès du parti, en octobre 1993, qui avait vu triompher le point de vue des «novateurs» (yenilikçiler) conduits par Recep Tayyip Erdogan qualifié de «musulman républicain», de «musulman libéral» ou encore de «musulman démocratique». Ce dauphin présumé du Président Erbakan à la tête du parti. Ce politicien islamiste nouvelle vague, qui avait fait adopter au congrès une plate forme plus séculière destinée à attirer de nouveaux groupes sociaux, assiste contrairement à Erbakan, aux cérémonies officielles du «Jour républicain» devant la statue d'Atatürk sur la place du Taksim à Istanbul. Il est aussi celui qui déclare publiquement : «Le nouvel an est célébré par les laïques et pas par nous... Je ne peux toutefois pas dire des laïques qu'ils ne sont pas des musulmans parce qu'ils fêtent le nouvel an. Seul Allah est habilité à juger en cette matière» ${ }^{38}$.

De toute évidence, le Parti de la Prospérité, devenu après son interdiction par la Cour constitutionnelle en 1998, le Parti de la Vertu (PV) ne présente pas - pas plus d'ailleurs que les autres mouvements ou confréries islamistes - un front monolithique. A côté d'une majorité de militants qui sont prêts à jouer le jeu démocratique et pluraliste, il existe des éléments doctrinaires et littéralistes, décidés à imposer une réislamisation radicale de la société par la contrainte partout où ils parviendraient au pouvoir. A ces éléments intransigeants il faut ajouter des groupuscules radicaux prêts à recourir à l'action violente pour imposer la création d'un État islamique. Ce noyau dur islamiste, comprenant des éléments plus ou moins violents était évalué à moins de $7 \%$ de la population.

Les activités de ces courants extrémistes sont apparemment mal perçues par la majorité des militants islamistes dans la mesure où elles donnent des arguments à l'establishment militaire pour dénoncer le danger islamiste et justifier les mesures prises à l'encontre des formations islamistes au nom de la défense de l'héritage kémaliste. Mais le ralliement de cette majorité de militants à l'action politique légale pour imposer leur modèle de société n'est pas seulement tactique, elle est l'expression d'une culture politique. Ce que veulent la plupart des militants du PP, souligne Rusen Cakir, c'est l'instauration d'une «théo-démocratie»; c'est l'implantation d'une théocratie par des voies démocratiques. Ces islamistes modérés aspirent, en fait, à une société islamique, non à unÉtat islamique; ils ne visent pas à une dé-laïcisation constitutionnelle et légale de l'État; ils n'exigent pas le rétablissement intégral de la sharia ${ }^{39}$. Si l'on peut souscrire à la définition que donne Rusen Cakir des aspirations des islamistes modérés, on ne peut manquer de relever ici la contradiction existant entre les concepts de théocratie et de démocratie.

Le premier concept, en effet, renvoie à la vision d'un État organisé par et autour des préceptes d'une révélation divine, tels qu'exposés dans le Coran et codifiés dans la sharia. Rien n'empêche pour autant l'existence au sein d'une théocratie de différents groupes politiques ou individus de tendances politiques différentes, ni que soient prévues dans un tel système des élections qui permettent aux électeurs de porter au pouvoir la tendance de leur choix. Une certaine forme d'alternance, qui est l'une des conditions essentielles de la démocratie, est donc possible à la condition de ne pas remettre en question le cadre d'obligations et de finalités issues de la révélation, dont les responsables politiques de l'État théocratique doivent assurer l'application. C'est le cas de l'Iran. Toutefois, le problème reste posé dans l'État islamique classique, où la religion fait la citoyenneté, du droit du non-musulman à participer à la gestion 
de la chose publique. Le fait pour un individu d'en être écarté en fonction de sa nonappartenance à la religion de la cité est en soi un principe non-démocratique. Ce problème ne se pose pas avec acuité en Turquie où la population est quasi exclusivement musulmane. Il n'en reste pas moins que la théocratie en excluant, dans son principe, le citoyen non-musulman du champ politique et des fonctions stratégiques dans l'État ne satisfait pas à la totalité des exigences de la démocratie.

Le second concept, quant à lui, implique que le choix exprimé par la société civile déterminera la composition d'un gouvernement et, le cas échéant, l'orientation, tant philosophique que politique, qu'il suivra. La société civile dans ce cas est entièrement responsable de ses choix politiques et de ses choix de société; aucune limite ne lui est, en théorie, imposée, par des individus, groupes ou religions, quant aux finalités qu'elle souhaite voir imprimées dans l'action de l'État. Une démocratie pleine et entière doit permettre à tout citoyen d'un État de participer, sans distinction de religion, à sa vie politique, et d'y occuper n'importe quelle charge en fonction de ses aspirations et de ses compétences. La liberté de conscience est ainsi inséparable de la notion de démocratie, et la laïcité de l'État en est l'une des expressions fondamentales.

Pour l'heure, les islamistes turcs modérés, parmi lesquels les intellectuels du mouvement déjà cités, n'ont apparemment pas encore accordé une attention particulière au problème que posait la contradiction fondamentale entre les notions de théocratie et de démocratie du point de vue du statut politique du non-musulman dans un État turc réislamisé.

A la lecture des écrits de la majorité des intellectuels et des prises de position publiques des novateurs du Parti de la Prospérité, devenu depuis 1998, le Parti de la Vertu, nous préférerons à la notion de «théo-démocratie» évoquée par Rusen Cakir, celles «d'islamisation de la société turque par des moyens démocratiques» ou de «société islamique pluraliste» citées par Metin Heper, dans la mesure où responsables politiques et penseurs du mouvement islamiste semblent vouloir préserver le système parlementaire représentatif comme source de légitimité du pouvoir et accepter les règles du jeu de la démocratie. Cette vision des choses se trouve confortée à la suite d'une série de rencontres effectuées avec une quarantaine de militants islamistes du Parti de la Prospérité d'Istanbul et une trentaine d'adhérents au parti d'Ankara en $1995^{40}$. A l'unanimité, nos interlocuteurs ont proclamé leur attachement à la démocratie parlementaire représentative dont l'avènement leur avait seul permis d'acquérir de la visibilité dans l'espace public et leur avait donné la possibilité d'accéder à l'expression politique de leurs aspirations et de leurs convictions. Tous se sont déclarés convaincus que seull'approfondissement de la démocratie peut garantir à l'avenir contre des interventions de l'armée aboutissant chaque fois à l'interdiction du parti islamiste comme ce fut le cas en 1972, 1980 et 1998. Interrogés sur leurs positions à propos du concept de liberté de conscience (Vicdan ou Inan özgürlügü), ils ont manifesté un certain embarras et, confondant apparemment les deux concepts, se sont lancés dans une défense et illustration argumentée de la tolérance (Hosgörü ou müsamaha) en islam. Poursuivant sur leur lancée, ils soulignaient, en particulier, leur rôle dans la démocratisation de la vie politique en Turquie, notamment au plan de l'action visant à entamer la vision dogmatique entretenue par l'establishment militaire et les kémalistes laïques radicaux d'une identité nationale monolithique. Les rapports de force étant ce qu'ils sont dans la société turque, nos interlocuteurs ne croyaient pas à la possibilité d'instaurer un régime islamique par la force. Euxmêmes estimaient que l'instauration d'un tel régime ne pouvait résulter que d'un souhait librement exprimé par le peuple, et déclaraient ne reconnaître comme légitime qu'un gouvernement issu d'élections libres. En ce sens, ils témoignent d'une culture politique qui les 
apparente globalement à leurs équivalents chrétiens d'Europe. L'accession du PP à un gouvernement de coalition en 1995, était de nature à les conforter dans leur comportement légaliste. La démission forcée du gouvernement de coalition PJV-PP présidé par Necmettin Erbakan, par contre, devrait les conforter dans l'idée que la lutte pour l'approfondissement de la démocratie n'est pas terminée pour eux.

Fait important dans le comportement légaliste des principaux représentants des courants islamistes modérés, ceux-ci semblent avoir opéré une analyse cohérente de l'état de la culture politique de la société turque globale. Au terme de leur analyse, confortée par plusieurs enquêtes notamment universitaires, il apparaît, comme nous l'avions déjà évoqué plus haut, que la majorité de l'électorat des partis islamistes qui ont été successivement représentés au Parlement, s'est focalisée sur les promesses et l'action sociale, plus que sur l'étiquette islamiste. Le vote de cette majorité d'électeurs, peu marqué par l'engagement idéologique, apparaissait dès lors principalement conditionné par le degré de satisfaction apporté par le parti aux attentes matérielles de la population. Le parti a donc logiquement conclu au caractère structurellement fluctuant de la majorité de son électorat; d'où la plate-forme électorale plus séculière, accordant une place prépondérante aux programmes sociaux, adoptée par le PP lors de son congrès de 1993.

Cette analyse des comportements électoraux turcs devait se révéler pertinente à la lumière des élections législatives d'avril 1999, qui ont été particulièrement éclairantes en ce qui concerne la volatilité actuelle de l'électorat turc. Le Parti de la Vertu (PV) héritier du PP n'obtenait qu'environ $16 \%$ des suffrages contre 21,5\% aux élections de 1994; ce parti passait de la première à la troisième place en ordre d'importance. A l'inverse le Parti du Mouvement National (PMN) passait de 8,2\% en 1995 à plus de $18 \%$ en 1999 , et, devenu le deuxième parti de Turquie, semblait avoir bénéficié d'un nombre non négligeable d'anciens électeurs du PP.

L'explication du recul du parti islamiste réside peut être dans l'insatisfaction éprouvée par un certain nombre d'électeurs à l'égard de la gestion municipale de certains élus du PP. En outre, le passage d'Erbakan à la tête du gouvernement de 1995 à 1997 n'a pas été particulièrement convainquant quant à sa capacité à changer significativement l'ordre des choses. En matière de politique étrangère, les gesticulations en direction des États islamistes en froid avec les ÉtatsUnis, n'ont pas entraîné de modification fondamentale en terme de système d'alliances. Dans ce domaine, comme dans les domaines économiques, les contraintes de la géopolitique et les impératifs sécuritaires qui en découlent, ajoutés aux poids des réalités économiques, rendent incontournable l'ancrage occidental et totalement utopique une éventuelle alternative fondée sur l'approfondissement des relations économiques et politiques avec les pays du MoyenOrient, de l'Asie Centrale et le monde musulman.

Si le succès remporté par le Parti Démocratique de la Gauche (PDG) peut s'expliquer comme un succès personnel de Bülent Ecevit, politicien intègre et compétent, dont la gestion des affaires avait été jugée satisfaisante, le succès remporté par le Parti du Mouvement National, qui, n'ayant même pas été représenté au Parlement, ne pouvait faire état d'aucun état de service dans la gestion de la chose publique, apparaît plutôt comme un vote de désaveu du système et de l'establishment politique.

Le succès remporté par le PMN, formation fondamentalement antidémocratique, dont l'agressivité a été quelque peu rabotée par le discours lénifiant de Devlet Bahçeli, successeur de Alparslan Türkes à la tête du parti, reste inquiétant dans la mesure où il n'a pas renoncé à ses credo fascistes. 
Ces élections débouchent d'une part sur un constat positif, d'autre part sur un constat potentiellement préoccupant.

Nous noterons sur le plan positif l'indice d'une maturité politique certaine, en ce sens que les votes ont majoritairement été émis par des individus de plus en plus conscients des enjeux politiques de leur choix et généralement motivés par une identification claire de leurs intérêts matériels, loin de toute motivation irrationnelle ou à caractère religieux. C'est ainsi, par exemple que les électeurs ont durement sanctionné les partis de centre-droit dont les chefs de file ont été compromis dans des affaires de corruption. Le PRP quant à lui a été désavoué en raison du manque d'envergure de ses dirigeants; ne passant pas la barre des $10 \%$ de suffrages nécessaires, il n'est même plus représenté à l'Assemblée Nationale.

Au plan négatif nous relèverons l'importance des suffrages accordés à un parti notoirement antidémocratique. Ce vote, qui apparaît essentiellement comme une manifestation de rejet, démontre combien la démocratie en Turquie demeure encore fragile. A cet égard l' accroissement des laissés pour compte du progrès économique et la progression des cas d'exclusion sociale sont autant de facteurs de nature à favoriser la progression de formations politiques qui remettent en cause le système actuel d'économie libérale et de démocratie parlementaire représentative, auquel manquent, entre autres, les dimensions de protection sociale et de démocratie économique susceptibles de les rendre désirables pour l'ensemble de la population. Ces carences présentent à terme, plus de dangers pour l'avenir de la démocratie en Turquie que l'islamisme en soi.

\section{CONCLUSION}

Sous l'effet de réformes culturelles, religieuses et institutionnelles radicales, menées à la hussarde par Mustafa Kemal Atatürk, le peuple turc a été coupé sans transition de ses racines et de tous ses systèmes de référence traditionnels. La quête d'identité qui en a résulté pour une large partie du corps social n'a toujours pas complètement abouti pour un certain nombre d'individus, même si de larges pans de la société turque ont aujourd'hui intégré les systèmes de référence de la démocratie parlementaire et des mécanismes institutionnels républicains.

De la même manière, on peut affirmer que les concepts d'État-Nation et de patriotisme sont aujourd'hui totalement intériorisés. «La socialisation en matière d'identité nationale a été progressivement réalisée, depuis les débuts de la République par l'intermédiaire des médias, de l'école, du salut au drapeau, par l'hymne national, par les parades d'État par les fêtes nationales non religieuses. Aujourd'hui le sentiment national, même si l'islam est ressenti par une partie importante du corps social comme une partie intégrante de son identité turque, prime le sentiment d'appartenance à la communauté musulmane» ${ }^{41}$.

Toutefois, si une identité nationale turque, volontiers ombrageuse, conforme aux vœux d'Atatürk, s'est forgée progressivement depuis l'instauration de la République, la laïcité, elle, continue à faire l'objet de réticences dans la mesure où elle est considérée par une partie importante de la population comme un élément de l'idéologie de l'establishment kémaliste et non comme un principe assurant la liberté de conscience. Par contre, sous les effets conjugués de l'environnement institutionnel, de la modernisation économique et de ses retombées sociales, du changement des conditions de vie et du cadre de références de l'ensemble de la population, la sécularisation des divers aspects de la vie privée et publique s'est opérée de manière progressive de la fondation de la République à la période actuelle, et constitue l'aboutissement d'un processus cumulatif. 
La remise en cause du principe kémaliste de laïcité représente donc moins une prise de position philosophique ou proprement religieuse, que l'opposition d'une partie de la population, principalement - mais pas uniquement ! - rurale frustrée, contre les représentants d'un ordre établi. Pour les groupes sociaux marginalisés, l'opposition islamique était un moyen d'exprimer leur opposition à un ordre considéré comme injuste et aliénant. L'introduction du pluralisme et la démocratisation progressive de la vie politique allait donner l'occasion aux organisations islamiques de canaliser, à leur profit, le mécontentent de larges fractions de l'opinion publique, notamment au travers d'actions de solidarité et de bienfaisance en faveur des laissés pour compte du développement économique libéral des années 1980-90. Toutefois, les progrès de l'islamisme ne peuvent en aucun cas être appréhendés seulement en termes de simple réaction à une situation de crise, mais aussi - et peut-être surtout - en termes de réappropriation de l'espace politique par ceux qui en étaient exclus. L'islamisme exprimerait ainsi à la fois une frustration, une volonté de réappropriation de la modernité. La montée politique des mouvements islamistes est donc un corollaire au développement de la société civile et au pluralisme en Turquie $^{42}$.

La Turquie est le seul exemple au Moyen-Orient - et même dans le monde musulman - où un tel processus de participation de formations islamistes à la vie politique a pu être mis en place par des moyens pacifiques et démocratiques. Et la manière dont la très grande majorité des groupes islamistes expriment leur différence et leur rejet de l'influence culturelle occidentale et des modes de vie et d'attitudes qu'elle suscite remet fondamentalement en question la vision qu'ont les nations occidentales, d'un islamisme violent par nature.

Dans la plupart des pays musulmans l'islamisme est né et s'est développé dans un cadre où les régimes en place ont été portés au pouvoir par la force et s'y maintiennent tant qu'ils continuent à s'assurer le monopole du pouvoir de coercition. L'absence de légitimité des détenteurs du pouvoir ne les autorisent pas à tolérer l'expression d'une opposition politique authentique sous peine d'être condamnés à la disparition. Tout au plus certains d'entre eux peuvent-ils, sous les pressions extérieures, s'autoriser un certain lifting destiné à donner l'illusion d'une vie parlementaire à l'occidentale. Dans cet environnement politique caractérisé par la violence d'État, seul le recours à l'action violente semble pouvoir permettre l'avènement d'un nouvel ordre politique et social. Le cas de l'Iran et de l'Algérie sont exemplaires à cet égard.

A l'inverse, comme seul semble le suggérer le cas de la Turquie, dans un environnement politique où la démocratie est devenue la norme du comportement politique, et où il est possible de soumettre librement ses choix de société aux suffrages des citoyens, les groupes islamistes - à l'exception de groupuscules extrémistes - ont apparemment renoncé définitivement à la violence pour accéder au pouvoir et à la contrainte pour s'y maintenir.

Si cet islam qui fait peur à l'Occident et qui rend improbable, aux yeux de nombreux citoyens européens, l'intégration de la Turquie dans une union d'États européens, a pu imposer sa visibilité dans l'espace public turc par des voies légales, c'est principalement parce que la culture politique du peuple turc a intégré les paramètres qui conditionnent l'exercice de la démocratie. En effet, pour la grande majorité des citoyens turcs - y compris les électeurs islamistes, le seul gouvernement considéré comme légitime est celui qui résulte d'élections libres et régulières. Ils partagent ainsi, aujourd'hui, avec les peuples d'Europe une culture politique commune. Par sa culture politique et sa culture économique, donc, la République turque s'apparente aux démocraties libérales européennes, même si, en raison de la vision 
jacobine et monolithique de l'État-Nation de l'establishment kémaliste littéraliste et doctrinaire, qui conduit à des manquements dans le domaine des droits de l'homme, la démocratie turque n'a pas encore intégré un certain nombre de standards propres aux démocraties européennes. Depuis la reconnaissance, par l'Europe, à Helsinki, de la recevabilité de la candidature turque à l'adhésion, un premier geste positif dans le sens d'une volonté d'approfondissement de la démocratie selon les critères européens, a été posé par la diplomatie turque en ce qui concerne le problème kurde.

Dans cette démocratie turque, où l'islam a conforté sa visibilité et sa réappropriation du champ politique, nombre de Turcs voient se confirmer la fin et l'échec du kémalisme.

Si l'on considère le kémalisme comme la reproduction littérale de l'ordre politique, économique, social et laïc instauré par Atatürk en son temps, alors il est indéniable que le kémalisme appartient au passé.

Si, par contre, il est conçu comme une démarche dynamique visant à approfondir de manière continue l'alignement de la Turquie sur les modèles institutionnels, politiques, économiques et sociaux offerts par les démocraties libérales européennes en évolution permanente, le kémalisme, comme élément de référence idéologique, garde une pertinence certaine.

\section{NOTES}

1 RIDA, Rashid, Le califa ou l'imâma suprême, traduit par Henri Laoust, Librairie d'Amérique et d'Orient, J. Maisonneuve, Paris, 1986, p. 116.

2 ABDUH, Muhammad, al-Islâm wa 'l-Nazrâniya ma'a 'l-'ilm wa 'l-Madâniya (L'islam et le christianisme face à la science et à la civilisation), le Caire, 1375 H/ 1960, pp. 60-62.

3 ABD AR_RAZIK, 'Alî, «al-Islâm wa usûl al-hukm» (l'islam et les bases de la souveraineté), Le Caire 1925, traduction française de BERCHER, dans Revue des Études islamiques, Paris 1933.

4 IBN TAYMIYYA, Siyâsa shar 'îya, traduit sous le titre Les statuts gouvernementaux de Ibn Taymiya, pp. 172-174.

5 LEWIS, Bernard, The Emergence of Modern Turkey, Oxford University Press, London, 1961, p. 318.

- KAZANCIGIL, Ali, «L'État laïque face à l'avancée de l'islam, dans Turquie : la nouvelle donne», Problèmes politiques et sociaux, $\mathrm{n}^{\circ} 757,1993$, pp. 13-14.

7 LEWIS, B. op. cit., pp. 323 - 324.

8 GÖLE, Nilüfer, Secularism and islamism in Turkey : the Making of Elites and Counter-Elites, Middle East Journal, vol. 51, n 1, Winter 1997.

9 KADIOGLU, Ayse, «The Paradox of Turkish Nationalism and the Construction of Official Identity» in Turkey : Identity, Democracy, Politics, Westview Press, Boulder, 1995, p. 88.

10 GÖLE, Nilüfer, «Démocratisation et poussée islamiste, dans Turquie : la nouvelle donne», Problèmes politiques et Sociaux, $\mathrm{n}^{\circ} 757,1993, \mathrm{p} .17$.

1 LEWIS, B., op. cit., pp. 412-414.

12 Cahiers de l'Orient Contemporain, 1947 et 1949.

13 Idem, 1950, 1951, 1953, 1955, 1959.

14 HEPER, Metin, «Islam, Politics and Society», Middle East Journal, Summer 1981, vol. 35, ${ }^{\circ} 3$, pp. 358-359.

15 HEPER, Metin, «Islam and Democracy in Turkey : towards a reconciliation», Middle East Journal, vol 51, $n^{\circ} 1$, Winter 1997, 35. 
16 KARPAT, Kemal, «Social Groups and the Political system after 1960», in Social Change and Politics in Turkey, E.J. Brill, Leiden, 1973, p. 31.

17 Idem, p. 232.

18 GÖLE, Nilüfer, «Secularism and islamism in Turkey : the Making of Elites and Counter-Elites», Middle East Journal, vol. 51, $\mathrm{n}^{\circ}$ 1, Winter 1997.

19 HEPER, Metin, «Islam, Politics and Society», op.cit., pp. 357-358.

20 DUMONT, Paul, «Le poids de l'islam en Turquie», L'Islam et l'État, dir. Olivier Carré, PUF, Paris 1982, p. 102.

21 DUMONT, Paul, «L'islam en Turquie : facteur de renouveau», dans Radicalisme islamique, Paris, l'Harmattan, 1986, pp. 368-371.

22 CAKIR, Rusen, Ne seriat, ne demokrasi (Ni sharia, ni démocratie), Siyahbeyaz Metis Güncel, Istanbul, 1994, p. 21.

23 DUMONT, Paul, «Le poids de l'islam en Turquie», op. cit., pp. 103-105.

24 GÖLE, Nilüfer, «Secularism and islamism», op. cit., p. 47.

25 Idem, p. 7.

26 HEPER, Metin, «Islam and Democracy in Turkey : towards Reconciliation», op. cit., p. 33.

27 BILICI, Faruk, «Islamistes de Turquie et d'Égypte : héritages communs, influences, stratégies articulées», dans La nouvelle dynamique au Moyen-Orient, L'Harmattan, Paris, 1993, pp. 3-36.

28 Idem, pp. 43-44.

29 HEPER, Metin, «Islam and Democracy in Turkey», op. cit., pp. 40-41.

30 GÖLE, Nilüfer, «The Making of Elites and Counter-Elites», op. cit., pp. 56-57.

31 HEPER, Metin, «Islam and Democracy in Turkey : Towards a Reconciliation», op. cit., p. 41.

32 GÖLE, Nilüfer, «The Making od Elites and Counter Elites», op. cit., p. 5.

33 HEPER, Metin, «Islam and Democracy : towards reconciliation», op. cit., p. 35.

34 Idem, p. 36.

35 AKINCI, Ugur, «The Municipal Radicalism of Political Islam in Turkey», Middle East Journal, vol. 53, n¹, Winter 1999, pp. 77-78.

36 Idem, pp. 79-81.

37 Idem, pp. 84-86.

38 HEPER, Metin, «Islam and Democracy : towards a Reconciliation», op. cit., p. 6.

39 CAKIR, Rusen, op. cit., pp. 128-130.

40 Brève enquête menée par nos soins en 1995 à Istanbul et Ankara sur la vision des militants islamistes d'un État conforme à leurs aspirations.

41 HEPER, Metin, «Islam, Politics and Society in Turkey», op cit., p. 355.

42 GOLE, Nilüfer, Démocratisation et poussée islamiste, op. cit. p. 17. 